\title{
Correction: Distinct co-acquired alterations and genomic evolution during TKI treatment in non-small-cell lung cancer patients with or without acquired T790M mutation
}

Ying Jin · Hua Bao · Xiuning Le - Xiaojun Fan · Ming Tang • Xun Shi • Jun Zhao · Junrong Yan • Yang Xu • Kelly Quek • Yasir Y. Elamin · Jianhua Zhang • P. Andrew Futreal • Ignacio I. Wistuba · John V. Heymach • Guangyuan Lou • Lan Shao - Qiong He $\cdot$ Chen Lin · Xue Wu · Yang W. Shao - Xiaonan Wang · Jiachen He - Yamei Chen • Justin Stebbing $\cdot$ Ming Chen · Jianjun Zhang $\cdot$ Xinmin Yu

Published online: 12 December 2019

(c) The Author(s), under exclusive licence to Springer Nature Limited 2019

\section{Correction to: Oncogene}

https://doi.org/10.1038/s41388-019-1104-z

The original version of this Article omitted the following from the Acknowledgements:

Professor Stebbing sits on SABs for Celltrion, Singapore Biotech, Vor Biopharma, TLC Biopharmaceuticals and
Benevolent AI, has consulted with Lansdowne partners, Vitruvian and Social Impact Capital and Chairs the Board of Directors for BB Biotech Healthcare Trust and Xerion Healthcare.

This has now been corrected in both the PDF and HTML versions of the Article. 